\title{
Utility of Endoscopic Ultrasound-Guided Hepaticogastrostomy with Antegrade Stenting for Malignant Biliary Obstruction after Failed Endoscopic Retrograde Cholangiopancreatography
}

\author{
Hajime Imai $^{a}$ Mamoru Takenaka $^{a}$ Shunsuke Omoto ${ }^{a}$ Ken Kamata ${ }^{a}$ \\ Takeshi Miyata $^{a}$ Kosuke Minaga ${ }^{a}$ Kentaro Yamao ${ }^{a}$ Toshiharu Sakurai ${ }^{a}$ \\ Naoshi Nishida ${ }^{a}$ Tomohiro Watanabe $^{a}$ Masayuki Kitano $^{b}$ Masatoshi Kudo $^{a}$ \\ a Department of Gastroenterology and Hepatology, Kindai University Faculty of Medicine, Osaka-Sayama, and \\ bSecond Department of Internal Medicine, Wakayama Medical University, Wakayama, Japan
}

\section{Keywords}

Endoscopic ultrasound-guided hepaticogastrostomy . Endoscopic ultrasound-guided antegrade stenting . Obstructive jaundice · Endoscopic ultrasound-guided biliary drainage

\begin{abstract}
Background: Endoscopic ultrasound (EUS)-guided biliary drainage $(B D)$ is a well-recognized alternative $B D$ method after unsuccessful endoscopic transpapillary drainage. EUSguided hepaticogastrostomy (HGS) with antegrade stenting (AGS) was recently applied to the treatment of malignant obstructive jaundice. Objective: To assess the efficacy and safety of HGS combined with AGS for treatment of malignant biliary stricture-induced obstructive jaundice. Design: Retrospective cohort study. Setting: Single academic tertiary care center. Patients: From January 2006 to December 2014, endoscopic retrograde cholangiopancreatography was at-
\end{abstract}

๑ 2017 S. Karger AG, Basel tempted in patients with obstructive jaundice; it was successful in 641 patients and impossible in 154 patients (postsurgically altered anatomy or duodenal stenosis, $n=101$; difficult cannulation, $n=53$ ). In total, 145 patients underwent EUS-guided BD; HGS and HGS with AGS were attempted in 42 patients (Group A, January 2006-August 2011) and 37 patients (Group B, September 2011-December 2014), respectively. Interventions: Under EUS and fluoroscopy guidance, HGS and HGS with AGS were performed via needle puncture, guidewire insertion, puncture-hole dilation, and stent placement. Main Outcome Measurements: Groups A and B were compared in terms of technical success, functional success, adverse event rates, re-intervention rates, patient survival time, and time to stent dysfunction or patient death. The two groups were also compared in a subgroup analysis of only 28 patients who underwent chemotherapy. Results: The technical success rate was significantly higher in Group A than $\mathrm{B}(97.6$ vs. $83.8 \%, p=0.03)$. The functional success rate was comparable between the two groups (90.2 vs. $90.3 \%$ ),

Dr. Mamoru Takenaka 
although the rate of adverse events was significantly higher in Group A than B (26.1 vs. $10.8 \%, p=0.03)$. The re-intervention rate tended to be higher in Group A than B (16.7 vs. 8.1\%, $p=0.25$ ). Groups $A$ and $B$ did not differ significantly in terms of median overall patient survival ( $75 \mathrm{vs} .61$ days, $p=0.70$ ) or median time to stent dysfunction or patient death ( $68 \mathrm{vs.} 63$ days, $p=0.08$ ). Among patients who underwent chemotherapy, there was no difference in overall patient survival time between the two groups (121 vs. 157 days, $p=0.08$ ), although time to stent dysfunction or patient death was significantly shorter in Group A than B (71 vs. 95 days, $p=0.02$ ). Conclusion: Although the technical success rate of HGS with AGS was lower than that of HGS, HGS with AGS was superior to HGS in terms of adverse event rate and stent patency in patients receiving chemotherapy.

(c) 2017 S. Karger AG, Basel

\section{Introduction}

The gold standard procedure for biliary drainage (BD) is transpapillary drainage during endoscopic retrograde cholangiopancreatography (ERCP). However, ERCP may not be possible in patients with an inaccessible biliary orifice, such as patients with a surgically altered anatomy or duodenal involvement of the tumor. Alternative drainage options in these patients include surgical biliary bypass or percutaneous transhepatic BD (PTBD); however, these procedures are associated with some adverse events [1, 2]. Endoscopic ultrasound (EUS)-guided BD (EUS-BD) techniques such as EUSguided choledochoduodenostomy (CDS), hepaticogastrostomy (HGS), antegrade stenting (AGS), and rendezvous stenting (RVS) have recently been shown to be useful for BD after unsuccessful ERCP [3-16]. Among these procedures, CDS and RVS require the echoendoscope to reach the duodenum. Therefore, HGS or AGS is indicated in patients with an inaccessible duodenum. However, HGS is associated with a higher risk of adverse events compared with the other methods [17]. When stent dysfunction occurs, re-intervention is more difficult after AGS alone than after HGS or CDS [18]. Thus, we began adding AGS during HGS in a single session in September 2011. The aim of this study was to evaluate the technical success, functional success, adverse events, re-intervention rate, and stent patency before (HGS alone) and after (HGS with AGS) combining both methods and to compare these outcomes between the two groups. The two groups were also compared only among patients receiving chemotherapy.

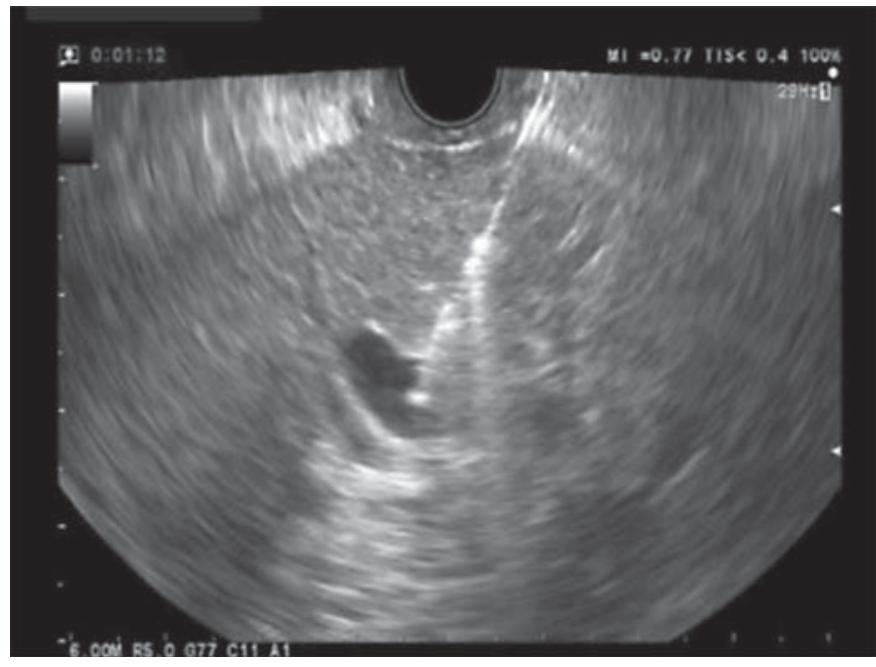

Fig. 1. Puncturing the intrahepatic bile duct. A $19-\mathrm{G}$ needle was inserted into the bile duct under endoscopic ultrasound guidance.

\section{Patients and Methods}

\section{Patients}

This study involved a retrospective analysis of data that had been prospectively collected from clinical records and imaging studies. In total, 795 patients were admitted to our hospital from January 2006 to December 2014 for treatment of obstructive jaundice caused by an unresectable malignant biliary stricture. In 88 patients, ERCP, CDS, and RVS were impossible because of an inaccessible duodenum caused by a surgically altered anatomy or duodenal involvement of the tumor. PTBD was performed in 9 patients. In the remaining 79 patients, we performed HGS with or without AGS. From January 2006 to August 2011, HGS alone was performed ( $n=42$, Group A). Beginning in September 2011, we added AGS during HGS in a single session ( $n=37$, Group B). The characteristics of patients of Groups A and B are shown in Table 1. All patients provided written informed consent to undergo HGS or HGS with AGS, and the study was approved by the ethics committee of our institution.

\section{Techniques}

HGS

An echoendoscope (GF-UCT240-AL5; Olympus, Tokyo, Japan) was introduced into the stomach and used to visualize the left hepatic bile duct. The scope was manipulated to identify an appropriate puncture route with no interposing vessels. A 19-G needle (SonoTip; Medi-Globe, Achenmühle, Germany) was inserted into the bile duct under EUS guidance. Bile was aspirated and contrast medium was injected into the bile duct for cholangiography. After confirmation of successful needle puncture by endosonographic and fluoroscopic imaging, a 0.035 -inch guidewire (RevoWave; Piolax, Yokohama, Japan) was introduced into the bile duct. The puncture tract was dilated by biliary dilation catheters $(6 \rightarrow 7 \rightarrow$ 9 Fr). A covered self-expandable metal stent (SEMS) was deployed between the left hepatic bile duct and the stomach.
Imai et al. 


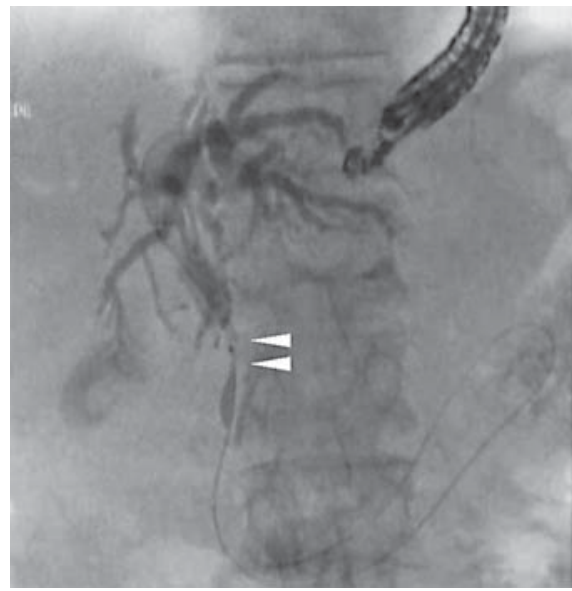

Fig. 2. Insertion of a guide wire. A 0.035inch guidewire was introduced into the bile duct and manipulated to pass the stricture (arrowheads). The duodenal and/or jejunal lumen was opacified with contrast material to direct the stent placement.

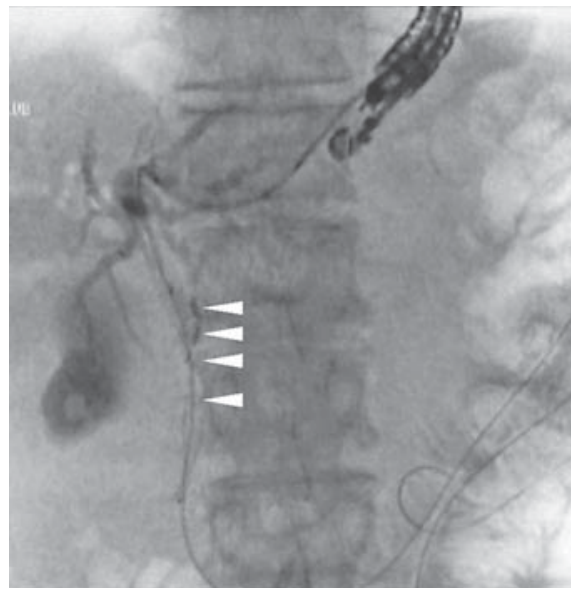

Fig. 3. Insertion of the stent at antegrade stenting. An uncovered self-expandable metal stent was deployed across the stricture (arrowheads).

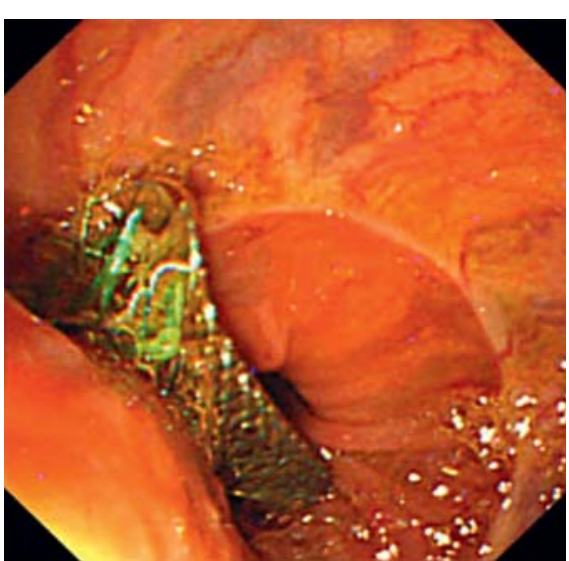

Fig. 4. Insertion of the stent at hepaticogastrostomy. A covered self-expandable metal stent was deployed between the left hepatic bile duct and the stomach.

Table 1. Characteristics and outcomes of the patients of Groups A and B

\begin{tabular}{lccc}
\hline & Group A $(n=42)$ & Group B $(n=37)$ & $p$ value \\
\hline Mean age \pm SD, years & $67.3 \pm 13.9$ & $77.0 \pm 11.5$ & 0.09 \\
Sex (male/female) & $24 / 18$ & $20 / 17$ & 0.79 \\
Primary disease, \% & & & \\
$\quad$ Pancreatic cancer & $31.0(13 / 42)$ & $24.3(9 / 37)$ & 0.51 \\
$\quad$ Bile duct cancer & $42.9(18 / 42)$ & $29.7(11 / 37)$ & 0.23 \\
$\quad$ Lymph node metastasis & $26.2(11 / 42)$ & $45.9(17 / 37)$ & 0.07 \\
Technical success rate, \% & 97.6 & 83.8 & 0.03 \\
Functional success rate, \% & 90.2 & 90.3 & 0.99 \\
Adverse events, \% & 26.1 & 10.8 & 0.03 \\
Re-intervention rate, \% & 16.7 & 8.1 & 0.25 \\
Mean procedure time \pm SD, min & $73.5 \pm 29.4$ & $70.1 \pm 30.9$ & 0.67 \\
Median overall survival (range), days & $75(5-354)$ & $61(16-335)$ & 0.70 \\
Median time to stent dysfunction or patient death (range), days & $68(5-185)$ & $63(16-335)$ & 0.08 \\
\hline
\end{tabular}

Groups A and B were compared using Fisher exact test, unpaired $t$ test, or log-rank test. From January 2006 to August 2011 , HGS alone was performed ( $n=42$, Group A). In September 2011, we introduced AGS before deployment of HGS in a single session ( $n=37$, Group B). SD, standard deviation; HGS, EUS-guided hepaticogastrostomy; AGS, EUS-guided antegrade stenting.

\section{HGS with AGS}

HGS with AGS is a modified HGS technique. An echoendoscope (GF-UCT240-AL5; Olympus, Tokyo, Japan) was introduced into the stomach and used to visualize the left hepatic bile duct. The scope was manipulated to identify an appropriate puncture route with no interposing vessels. A 19-G needle (SonoTip; MediGlobe, Achenmühle, Germany) was inserted into the bile duct under EUS guidance (Fig. 1). Bile was aspirated and contrast medium was injected into the bile duct for cholangiography. After confirmation of successful needle puncture by endosonographic and fluoroscopic imaging, a 0.035-inch guidewire (RevoWave, Piolax, Yokohama, Japan) was introduced into the bile duct and manipulated to pass the stricture. The duodenal and/or jejunal lumen was opacified with contrast material to direct the stent placement (Fig. 2). An uncovered SEMS (Zeo Stent; ZEON Medical, Tokyo, Japan) was deployed across the stricture (AGS) (Fig. 3). After AGS, the puncture tract was dilated using biliary dilation catheters $(6 \rightarrow 7 \rightarrow 9 \mathrm{Fr})$ (Soehendra Biliary Dilation Catheter; Cook Medical, Bloomington, IN, USA). A covered SEMS was deployed between the left hepatic bile duct and the stomach (Fig. 4). 
Table 2. Characteristics and outcomes of the patients of Groups A and B in chemotherapy

\begin{tabular}{lccc}
\hline & Group A $(n=14)$ & Group B $(n=14)$ & $p$ value \\
\hline Mean age \pm SD, years & $70.6 \pm 6.2$ & $66.1 \pm 10.0$ & 0.17 \\
Sex (male/female) & $7 / 7$ & $9 / 5$ & 0.45 \\
Primary disease, $\%$ & & $7.1(1 / 14)$ & 0.07 \\
$\quad$ Pancreatic cancer & $35.7(5 / 14)$ & $35.7(5 / 14)$ & 1.0 \\
$\quad$ Bile duct cancer & $35.7(5 / 14)$ & $57.1(8 / 14)$ & 0.13 \\
$\quad$ Lymph node metastasis & $28.6(4 / 14)$ & 14.2 & 0.19 \\
Adverse events, \% & 35.7 & 7.1 & 0.13 \\
Re-intervention rate, \% & 28.6 & $74.4 \pm 6.6$ & 0.09 \\
Mean procedure time \pm SD, min & $59.5 \pm 5.4$ & $157(52-390)$ & 0.08 \\
Median overall survival (range), days & $121(68-354)$ & $95(16-335)$ & 0.02 \\
Median time to stent dysfunction or patient death (range), days & $71(58-185)$ & \\
\hline
\end{tabular}

Groups A and B were compared using Fisher exact test, unpaired $t$ test, or log-rank test. Between January 2006 and August 2011, HGS alone was performed ( $n=14$, Group A). In September 2011, we introduced AGS before deployment of HGS in a single session ( $n=14$, Group B). SD, standard deviation; HGS, EUS-guided hepaticogastrostomy; AGS, EUS-guided antegrade stenting.

\section{Outcome Assessment}

The assessed outcomes were the technical and functional success rates, adverse event rate, re-intervention rate, procedure time, overall patient survival time, and time to stent dysfunction or patient death. In Group A, technical success of HGS was defined as successful stent deployment between the left hepatic bile duct and the stomach. In Group B, technical success of HGS with AGS was defined as successful stent deployment at the bile duct stricture (AGS) in addition to successful HGS. Functional success for obstructive jaundice was defined as a decrease in the bilirubin concentration to $<40 \%$ of the pretreatment value within 2 weeks. The incidence rate of adverse events such as peritonitis, bile leakage, bleeding, stent migration, and stent occlusion was also assessed. Re-intervention was defined as any endoscopic, surgical, or percutaneous procedure that was required to improve symptoms after placement of the stent. The time to stent dysfunction or patient death was defined as the time from stent deployment to biliary reintervention due to stent dysfunction or the time from stent deployment to patient death. Groups A and B were compared in terms of technical success, clinical success, adverse event outcomes, procedure time, overall patient survival, and time to stent dysfunction or patient death. For the subgroup analysis of only patients who underwent chemotherapy, Groups A and B were compared in terms of overall patient survival and the time to stent dysfunction or patient death.

\section{Statistical Analysis}

Statistical analysis was performed using SPSS version 11.0 (SPSS Inc., Chicago, IL, USA). Continuous variables were compared between the two groups using Student $t$ test. The rates of success, adverse events, and re-intervention were compared between the two groups using Fisher exact test. The Kaplan-Meier method was used to measure overall patient survival and the time to stent dysfunction or patient death. The log-rank test was used to compare Groups A and B in terms of overall patient survival and the time to stent dysfunction or patient death. A two-tailed $p$ value of $<0.05$ was considered statistically significant.

\section{Results}

The technical success rate was significantly higher in Group A than B (97.6 vs. 83.8\%, $p=0.03$ ) (Table 1). In Group A, deployment of the covered SEMS between the bile duct and stomach was unsuccessful in only 1 patient. In Group B, AGS failed because a guidewire did not pass the stricture in 6 patients, although the HGS technique was successful in all patients. Functional success was achieved in 37 patients (90.2\%) in Group A and 28 patients $(90.3 \%)$ in Group B. There was no significant difference in the functional success rate between Groups A and $\mathrm{B}(p=0.99)$.

Re-intervention was required in 7 (16.7\%) and 3 (8.1\%) patients in Groups A and B, respectively $(p=0.25)$. There was no significant difference between Groups A and B in terms of the re-intervention rate, although the rate was higher in Group A. The mean procedure times were 73.4 \pm 29.4 and $70.1 \pm 30.9 \mathrm{~min}$ in Groups $\mathrm{A}$ and $\mathrm{B}$, respectively $(p=0.67)$. In Group A, 11 patients $(26.2 \%)$ had procedural-related adverse events including bile leakage $(16.7 \%, n=7)$, stent migration $(4.8 \%, n=2)$, and cholangitis $(4.8 \%, n=2)$. In Group B, 4 patients $(10.8 \%)$ had procedural-related adverse events including mild acute pancreatitis $(5.4 \%, n=2)$, bile leakage $(2.7 \%, n=1)$, and cholangitis $(2.7 \%, n=1)$. Two patients with stent migration in Group A required PTBD. All other patients were managed conservatively. There were significant differences in the adverse event rates between Groups A and B $(p=0.03)$. At the time of the retrospective evaluation (June 30, 2015), all patients had died. The median sur- 
Fig. 5. The Kaplan-Meier curves for the overall patient survival time in Groups A and $\mathrm{B}$ among patients who underwent chemotherapy. The median survival times in Groups A and B among patients receiving chemotherapy were 121 and 157 days, respectively ( $p=0.08, \log$-rank test).
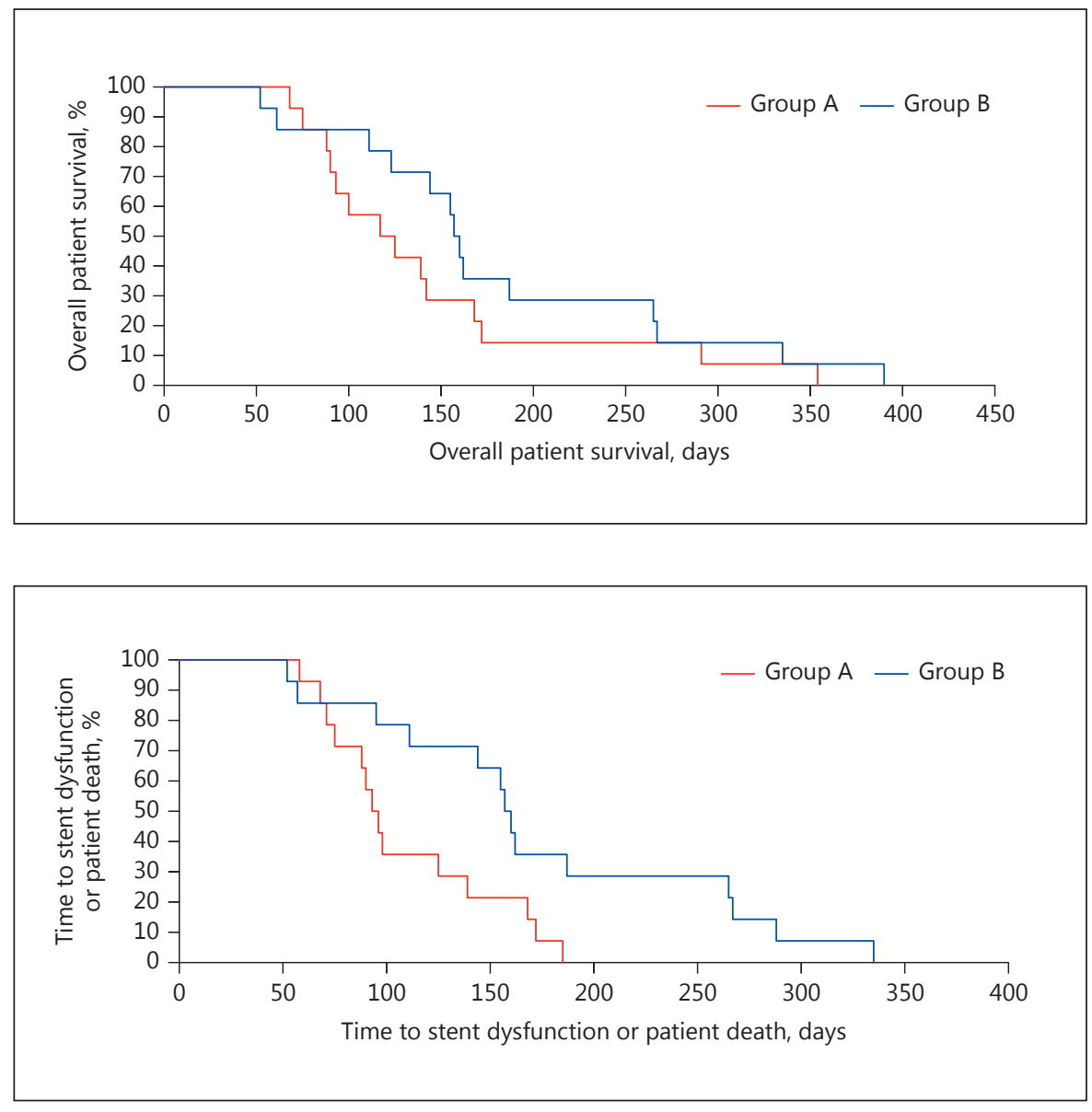

Fig. 6. The Kaplan-Meier curves for the median time to stent dysfunction or patient death in Groups A and B among patients who underwent chemotherapy. The median time to stent dysfunction or patient death in Groups A and B among patients receiving chemotherapy were 71 and 95 days, respectively ( $p=0.02, \log$-rank test).

vival times in Groups A and B were 75 and 61 days, respectively $(p=0.70)$. The median time to stent dysfunction or patient death in Groups A and B were 68 and 63 days, respectively $(p=0.08)$. Groups A and B did not differ significantly in terms of overall patient survival or time to stent dysfunction or patient death. Table 2 shows the detailed characteristics of the patients who underwent chemotherapy. There were no differences in any outcomes between Groups A and B, although the re-intervention rate tended to be higher in Group A than B (28.6 vs. $7.1 \%, p=0.13$ ). Figures 5 and 6 show the Kaplan-Meier curves for the overall patient survival time and time to stent dysfunction or patient death in Groups A and B among patients who underwent chemotherapy. The median survival times in Groups A and B among patients receiving chemotherapy were 121 and 157 days, respectively $(p=0.08)$. The median time to stent dysfunction or patient death in Groups A and B among patients receiving chemotherapy were 71 and 95 days, respectively $(p=$ 0.02).

EUS-Guided Hepaticogastrostomy with Antegrade Stenting

\section{Discussion}

In 2001, Giovannini et al. [3] published the first report of EUS-guided biliary access from the duodenal bulb followed by plastic stent placement after failed ERCP. Several EUS-BD methods such as CDS, HGS, AGS, and RVS were subsequently reported with respect to the utility of BD methods after unsuccessful ERCP [4-12]. These reports concluded that CDS is indicated in cases of distal biliary obstruction. However, CDS is sometimes difficult to perform because the duodenum is inaccessible due to tumor invasion or after surgical reconstruction. In such cases, HGS or AGS is regarded as the preferred method. Although the choice of transluminal drainage does not appear to affect the adverse event rate, HGS is associated with a higher risk of adverse events [17].

HGS with AGS may have an advantage with respect to adverse events, re-intervention method, and stent patency. HGS with AGS may prevent or reduce adverse events because AGS is carried out without dilation of the HGS

Oncology 2017;93(suppl 1):69-75 DOI: $10.1159 / 000481233$ 
fistula and prevents bile leakage during the dilation of the fistula [18]. Indeed, our results showed that the adverse event rate in Group B (10.8\%) is significant lower than that in Group A (26.2\%). In particular, the rate of bile leakage in Group B (2.7\%) was lower than that in Group A (14.3\%). However, mild acute pancreatitis occurred in 2 patients with obstructive jaundice caused by lymph node metastasis of gastric cancer in Group B. A stent deployed not only at the stricture but also at the major papilla may cause acute pancreatitis by compression of the pancreatic duct orifice. Another study using AGS also showed acute pancreatitis after treatment in $8.3 \%$ of patients [18]. These results suggest that we must consider the possibility of acute pancreatitis after AGS and avoid stent deployment at the papilla.

If stent occlusion occurs in patients who have undergone AGS alone, re-intervention is very difficult. However, re-intervention can be easily performed through the HGS stent in patients who have undergone HGS with AGS [18]. Indeed, we placed the plastic stent through the HGS stent in 3 patients (Group A, $n=1$; Group B, $n=2$ ) in whom stent occlusion occurred.

Another reason to perform HGS with AGS is that longer stent patency might be obtained than with HGS or AGS only. When one stent becomes occluded, the other stent offers an outlet and avoids obstructive jaundice. One report described stent dysfunction between CDS and HGS [19]. According to that study, stent occlusion resulted in a higher dysfunction rate in HGS than CDS. However, data regarding stent patency in EUS-BD is lacking because of short follow-up periods and survival times. In the present study, Groups A and B did not differ sig- nificantly in terms of the median time to stent dysfunction or patient death ( 68 vs. 63 days, $p=0.08$ ), but the median time to stent dysfunction or patient death among patients receiving chemotherapy was longer in Group B than A (95 vs. 71 days, $p=0.02$ ).

The technical success rate was lower in Group B than A in the current study. The technique of HGS with AGS is similar to that of transgastric RVS. In previous reports about transgastric RVS, the technical success rate ranged from 44 to $100 \%$ [20-24]. The most frequent reason for failure of RVS was difficulty in passing the guidewire through the stricture. In our study, 6 cases were attributed to failure to pass a guidewire through the stricture. In these cases, we decided during the procedure to perform HGS.

Our study has two limitations: its retrospective design and performance at a single center. The retrospective nature of the study could have allowed for potential bias in patient selection, limiting the validity of the conclusions. Because the study was conducted at an expert center, the results may not apply universally.

In conclusion, this study shows that EUS-HGS both with and without AGS has equivalent short-term safety and efficacy when performed by experts. However, HGS plus AGS is preferred in patients undergoing chemotherapy because it allows for longer stent patency.

\section{Disclosure Statement}

The authors have no conflicts of interest to declare.

\section{References}

1 Smith AC, Dowsett JF, Russell RC, et al: Randomised trial of endoscopic stenting versus surgical bypass in malignant low bile duct obstruction. Lancet 1994;344:1655-1660.

2 Stoker J, Lameris JS: Complications of percutaneously inserted biliary Wallstents. J Vasc Interv Radiol 1993;4:767-772.

3 Giovannini M, Moutardier V, Pesenti C, et al: Endoscopic ultrasound-guided bilioduodenal anastomosis: a new technique for biliary drainage. Endoscopy 2001;33:898-900.

4 Will U, Thieme A, Fueldner F, et al: Treatment of biliary obstruction in selected patients by endoscopic ultrasonography (EUS)guided transluminal biliary drainage. Endoscopy 2007;39:292-295.
5 Burmester E, Niehaus J, Leineweber T, et al: EUS-cholangio-drainage of the bile duct: report of 4 cases. Gastrointest Endosc 2003;57: 246-251.

6 Kim YS, Gupta K, Mallery S, et al: Endoscopic ultrasound rendezvous for bile duct access using a transduodenal approach: cumulative experience at a single center. A case series. Endoscopy 2010;42:496-502.

7 Siddiqui AA, Sreenarasimhaiah J, Lara LF, et al: Endoscopic ultrasound-guided transduodenal placement of a fully covered metal stent for palliative biliary drainage in patients with malignant biliary obstruction. Surg Endosc 2011;25:549-555.
8 Hara K, Yamao K, Niwa Y, et al: Prospective clinical study of EUS-guided choledochoduodenostomy for malignant lower biliary tract obstruction. Am J Gastroenterol 2011;106: 1239-1245.

9 Komaki T, Kitano M, Sakamoto H, et al: Endoscopic ultrasonography-guided biliary drainage: evaluation of a choledochoduodenostomy technique. Pancreatology 2011; 11(suppl 2):47-51.

10 Dhir V, Bhandari S, Bapat M, et al: Comparison of EUS-guided rendezvous and precut papillotomy techniques for biliary access (with videos). Gastrointest Endosc 2012;75:354359. 
11 Vila JJ, Perez-Miranda M, Vazquez-Sequeiros E, et al: Initial experience with EUS-guided cholangiopancreatography for biliary and pancreatic duct drainage: a Spanish national survey. Gastrointest Endosc 2012;76:11331141.

12 Nguyen-Tang T, Binmoeller KF, SanchezYague A, et al: Endoscopic ultrasound (EUS)guided transhepatic anterograde self-expandable metal stent (SEMS) placement across malignant biliary obstruction. Endoscopy 2010;42:232-236.

13 Artifon EL, Safatle-Ribeiro AV, Ferreira FC, et al: EUS-guided antegrade transhepatic placement of a self-expandable metal stent in hepatico-jejunal anastomosis. JOP 2011;12: 610-613.

14 Weilert F, Binmoeller KF, Marson F, et al: Endoscopic ultrasound-guided anterograde treatment of biliary stones following gastric bypass. Endoscopy 2011;43:1105-1108.
15 Park DH, Jang JW, Lee SS, et al: EUS-guided transhepatic antegrade balloon dilation for benign bilioenteric anastomotic strictures in a patient with hepaticojejunostomy. Gastrointest Endosc 2012;75:692-693.

16 Iwashita T, Yasuda I, Doi S, et al: Endoscopic ultrasound-guided antegrade treatments for biliary disorders in patients with surgically altered anatomy. Dig Dis Sci 2013;58:24172422.

17 Dhir V, Artifon EL, Gupta K, et al: Multicenter study on endoscopic ultrasound-guided expandable biliary metal stent placement: choice of access route, direction of stent insertion, and drainage route. Dig Endosc 2014;26: 430-435.

18 Ogura T, Masuda D, Imoto A, et al: EUSguided hepaticogastrostomy combined with fine-gauge antegrade stenting: a pilot study. Endoscopy 2014;46:416-421.

19 Hara K, Yamao K, Mizuno N, et al: Endoscopic ultrasonography-guided biliary drainage: who, when, which, and how? World J Gastroenterol 2016;22:1297-1303.
20 Artifon EL, Marson FP, Gaidhane M, et al: Hepaticogastrostomy or choledochoduodenostomy for distal malignant biliary obstruction after failed ERCP: is there any difference? Gastrointest Endosc 2015;81:950-959.

21 Iwashita T, Lee JG, Shinoura S, et al: Endoscopic ultrasound-guided rendezvous for biliary access after failed cannulation. Endoscopy 2012;44:60-65.

22 Maranki J, Hernandez AJ, Arslan B, et al: Interventional endoscopic ultrasoundguided cholangiography: long-term experience of an emerging alternative to percutaneous transhepatic cholangiography. Endoscopy 2009; 41:532-538.

23 Kahaleh M, Hernandez AJ, Tokar J, et al: Interventional EUS-guided cholangiography: evaluation of a technique in evolution. Gastrointest Endosc 2006;64:52-59.

24 Kawakubo K, Isayama H, Sasahira N, et al: Clinical utility of an endoscopic ultrasoundguided rendezvous technique via various approach routes. Surg Endosc 2013;27:34373443. 